\title{
Outcome of management of brain abscess in children
}

\author{
Ikram Ullah Khan ${ }^{1}$, Abdul Latif ${ }^{2}$, Muhammad Ashraf ${ }^{3}$, \\ Muhammad Kashif Chishti ${ }^{4}$, Sadia Sadiq ${ }^{5}$
}

\begin{abstract}
Objective: To find out the outcome of management of brain abscess in children.

Methods: This is prospective observational study conducted in the Department of Paediatric Neurosurgery at Children's Hospital and Institute of Child Health, Multan from July 2014 to June 2017. Children up to the age of 14 years suffering from brain abscess were admitted. After taking clinical history, general and systemic physical examination and necessary investigations, abscess was evacuated and abscess wall excised after performing craniotomy. Data was collected on a predesigned performa. Results were analyzed and compared with national and international literature through statistical package for social sciences (SPSS-20).

Results: Twenty five patients up to 14 years of age were included. Seventeen $(68 \%)$ were male and eight (32\%) female. Fever and vomiting were present in all $25(100 \%)$ patients. Paranasal sinusitis was predisposing causative factor in $9(36 \%)$ followed by otitis media in $7(28 \%)$. Abscess was present in frontal lobe in $9(36 \%)$, temporoparietal region in $8(32 \%)$, posterior fossa in $5(20 \%)$ and multiple abscesses in 3 $(12 \%)$. Craniotomy was performed, pus evacuated and abscess wall excised in all 25 (100\%) patients. Three (12\%) patients expired.

Conclusion: Incidence of brain abscess can be decreased by treatment of its predisposing causes as sinusitis and otitis media. Small abscess less than $2 \mathrm{~cm}$ can be treated with antibiotics. Complete evacuation of pus and excision of abscess wall after performing craniotomy along with appropriate antibiotics is gold standard management of brain abscess in children.
\end{abstract}

KEYWORDS: Brain abscess, Children, Craniotomy.

doi: https://doi.org/10.12669/pjms.36.3.1087

How to cite this:

Khan IU, Latif A, Ashraf M, Chishti MK, Sadiq S. Outcome of management of brain abscess in children. Pak J Med Sci. 2020;36(3):306-309. doi: https://doi.org/10.12669/pjms.36.3.1087

This is an Open Access article distributed under the terms of the Creative Commons Attribution License (http://creativecommons.org/licenses/by/3.0), which permits unrestricted use, distribution, and reproduction in any medium, provided the original work is properly cited.

1. Ikram Ullah Khan,

Assistant Professor, Department of Paediatric Surgery,

2. Abdul Latif,

Associate Professor, Department of Paediatric Surgery,

Nishtar Medical University, Multan, Pakistan.

3. Muhammad Ashraf,

Associate Professor, Department of Paediatric Neurosurgery,

4. Muhammad Kashif Chishti

Professor of Paeds: Surgery,

5. Sadia Sadiq,

Department of Radiology,

1,3-5:The Children's Hospital \& The Institute of Child Health, Multan, Pakistan.

Correspondence:

Dr. Ikram Ullah Khan,

Assistant Professor, Department of Paediatric Surgery,

The Children's Hospital \& The Institute of Child Health,

Multan, Pakistan.

E-mail: doctorikram2007@gmail.com

* Received for Publication:

May 18, 2019

* Revision Received:

* Accepted for Publication:

February 6, 2020

\section{INTRODUCTION}

Brain abscess is defined as focal suppurative process within the brain parenchyma that begins as a localized area of cerebritis and changes into collection of pus surrounded by a well vascularized capsule. Infectious process that affects the CNS may threaten vital neurological functions and even life itself. The prognosis of these patients has improved over the past 25 years in large measure due to technological advances in diagnostic and treatment modalities as new generation of antibiotics, CT and MRI. However, in spite of these advances, CNS infections continue to add to neurological morbidity and mortality. ${ }^{1}$

The common predisposing factors are paranasal sinusitis, otitis media, trauma, brain surgery and 
cyanotic heart diseases. Mode of entry of causative microorganism is contiguous spread from infected paranasal sinuses and otitis media, hematogenous in endocarditis due to cyanotic heart diseases and direct inoculation in brain during trauma and surgery. ${ }^{2}$ Fever, headache, vomiting, seizures are common presentations. Altered level of consciousness, neck rigidity and focal neurological deficits may also be present. $^{3}$ Intraventricular rupture of abscess and brain herniation may be fatal complications. An abscess has a stereotyped appearance and ring enhancement on contrast enhanced CT. ${ }^{4}$ Diffusion waited MRI may be helpful in diagnosing brain abscess even at cerebritis stage and differentiating it from other cystic brain lesions. ${ }^{5}$ Small abscess and cerebritis stage respond to parenteral antibiotics and may be treated completely with medical management only without any surgical intervention. ${ }^{6}$ Surgical treatment of brain abscess is aspiration of pus or craniotomy and excision of abscess followed by antibiotic therapy. ${ }^{7}$ Burr hole is made at Kocher's point for abscess in frontal lobe, Keen's point for temporoparietal region and at Frasier's point for occipital region. Craniotomy was performed according to location of abscess.

Choice of surgical procedure depends upon surgeon and is debatable. Aspiration of pus is minimal invasive technique and can be repeated as well, while craniotomy and excision of abscess is an extensive surgical procedure requires long anesthesia. ${ }^{8}$ This study was carried out with an objective to assess the outcome of various surgical options of management of brain abscess.

\section{METHODS}

This is prospective observational study conducted in the Department of Paediatric Neurosurgery at Children's Hospital and Institute of Child Health Multan for a period of three years from July 2014 to June 2017 after approval from ethical committee dated February 26, 2019. All the patients suffering from brain abscess were admitted throughout patient department or referred from department of paediatric medicine of these hospitals. After taking history and clinical examination, necessary investigations like complete blood cells count and CT scan brain plain and with contrast were performed. MRI was performed if needed. Patients in cerebritis stage of disease or small abscess less than $2 \mathrm{~cm}$ without causing mass effect were referred to respective department of paediatric medicine for conservative medical management and not included in this study.
Surgical intervention was planned in those patients having brain abscess with size more than $2 \mathrm{~cm}$, causing mass effects neurological deficit, at aliquant area or with signs of raised intracranial pressure and these patients were included in the study. Mannitol $(20 \%)$ was used if signs of raised intracranial pressure were present. Meropenum, vancomycin and metronidazole were started intravenously. Patients were assessed for fitness for general anesthesia and surgical intervention. Pus was aspirated with needle through open anterior fontanelle in infant age group. A burr hole was made at appropriate site to aspirate pus with brain cannula in children above one year of age. Craniotomy for excision of abscess was performed in case of thick loculated septated pus not aspirated through burr hole. In case of multiple abscesses, only larger size abscesses were aspirated. Pus was sent for culture and sensitivity and antibiotics were changed accordingly. Check scan was performed after one week if clinical features of recollection of abscess appeared during the course of therapy. Intravenous antibiotics were continued for 4 to 6 weeks and shifted to oral rout for another. Four to six weeks depending upon clinical and radiological response. Follow-up CT scan was obtained at the completion of therapy routinely and in between if required.

Statistical analysis: Data was collected on a pre-designed performa and analyzed through statistical package for social sciences (SPSS-20). The results were compared with national and international literature.

\section{RESULTS}

A total of 25 patients suffering from brain abscess were managed. Seventeen $(68 \%)$ were male and $8(32 \%)$ female. Age range was up to 14 years

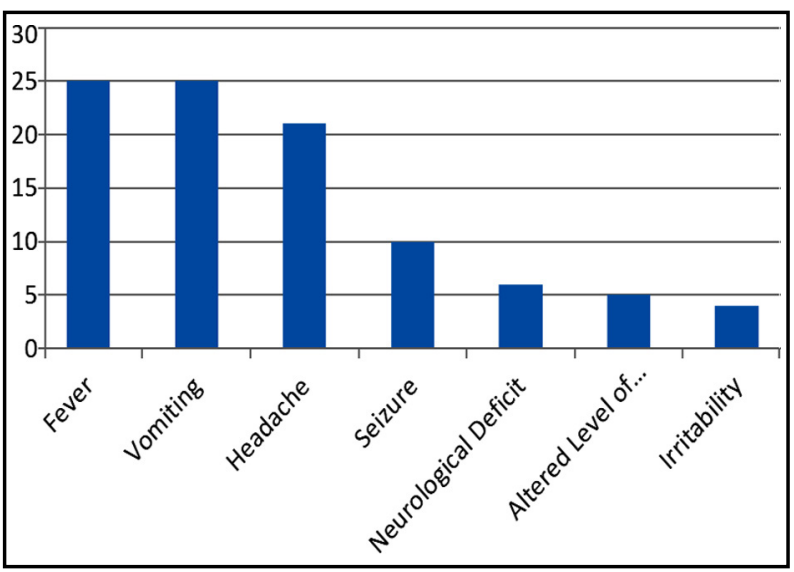

Fig.1: Clinical presentation of Children with Brain Abscess $(\mathrm{N}=25)$. 
Table-I: Predisposing causative factor.

\begin{tabular}{lcc}
\hline Causative factor & No. of Patients & Percentage \\
\hline Paranasal sinusitis & 9 & $36 \%$ \\
Otitis media & 7 & $28 \%$ \\
Endocarditis & 4 & $16 \%$ \\
Brain trauma & 2 & $8 \%$ \\
Unknown & 3 & $12 \%$ \\
\hline Total & 25 & $100 \%$ \\
\hline
\end{tabular}

including four infants. Fever and vomiting were present in all $25(100 \%)$ patients, headache in 21 $(84 \%)$ seizure in $10(40 \%)$ focal neurological deficit in $6(24 \%)$ and irritability in $4(16 \%)$ patients.

Paranasal sinusitis was predisposing causative factor in $9(36 \%)$, otitis media in $7(28 \%)$, endocarditis secondary to cyanotic heart disease in $4(16 \%)$, brain trauma in $2(8 \%)$ and in $3(12 \%)$ patients no causative factor identified.

Abscess was present in frontal lobe in $9(36 \%)$, temporoparietal region in $8(32 \%)$, posterior fossa in $5(20 \%)$ and multiple abscess in $3(12 \%)$.

Pus was aspirated with needle through open anterior fontanelle in $4(16 \%)$ patients less than one year of age, with brain cannula after making a burr hole in $16(60 \%)$ patients and craniotomy performed for excision of thick multiloculated septated pus in $6(24 \%)$ patients. Three $(12 \%)$ patients expired, two after craniotomy and one after aspiration of abscess.

\section{DISCUSSION}

This study was performed in children age group up to 14 years. A total of 25 patients suffering from brain abscess were managed and included in the study. Twenty-one children were between 1-14 years of age and four were infants under one year of age. Seventeen $(68 \%)$ were male and $8(32 \%)$ female with male to female ratio 2:1. 9. Muzumdar D et al. mentioned that brain abscess can develop at any age, $71 \%$ in male and $29 \%$ in female with male to female ratio of almost 2:1. ${ }^{9}$ Ranjith et al. mentioned it to be more common in younger age group mostly in first three decades. ${ }^{7}$

Fever and vomiting were present in all $25(100 \%)$ patients, headache in $21(84 \%)$ seizure in $10(40 \%)$,

Table-II: Region of location of brain abscess

\begin{tabular}{lcc}
\hline Location area & No. of Patients & Percentage \\
\hline Frontal lobe & 9 & $36 \%$ \\
Temporoparietal & 8 & $32 \%$ \\
Posterior fossa & 5 & $20 \%$ \\
Multiple abscess & 3 & $12 \%$ \\
\hline Total & 25 & $100 \%$ \\
\hline
\end{tabular}

Table-III: Treatment modalities.

\begin{tabular}{lcc}
\hline Treatment modality & No. of Patients & Percentage \\
\hline $\begin{array}{c}\text { Needle aspiration } \\
\text { through fontanelle }\end{array}$ & 4 & $16 \%$ \\
$\begin{array}{c}\text { Burr hole aspiration } \\
\text { with brain cannula }\end{array}$ & 15 & $60 \%$ \\
$\begin{array}{c}\text { Craniotomy and } \\
\text { excision of abscess }\end{array}$ & 6 & $24 \%$ \\
\hline
\end{tabular}

focal neurological deficit in $6(24 \%)$ and altered level of conciseness in $5(20 \%)$. Four patients were infants unable to speak presented with irritability and reluctant to feed. Eva Tonon et al. reported fever in $79 \%$, headache in $69 \%$, neurological deficit in $66 \%$, seizer in $27 \%$ and altered level of conciseness in (21\%). ${ }^{10}$ Chuang et al. mentioned seizers after bacterial brain abscess and outcome of its management. ${ }^{11}$

Paranasal sinusitis was predisposing causative factor in 9(36\%), otitis media in $7(28 \%)$, endocarditis secondary to cyanotic heart disease in $4(16 \%)$, brain trauma in $2(8 \%)$ and in $3(12 \%)$ no underlying cause was found. Muzumdar D et al. also mentioned paranasal sinusitis as a leading predisposing factor followed by otitis media. ${ }^{9}$ Brain abscess without any known underlying cause has been reported by Stanescu GL et al. ${ }^{12}$ Brain abscess in immune compromised patient was not noted in this study but it is reported in literature by Nelson et al. ${ }^{13}$ Similarly abscess may develop after brain surgery as mentioned by Yang et al. ${ }^{14}$, but not noted in this study. Abscess was present in frontal lobe in 9 (36\%), temporoparietal region in $8(32 \%)$, posterior fossa in $5(20 \%)$ and multiple abscess in $3(12 \%)$. Eva Tonon et al. reported location of abscess in temporal lobe $36 \%$, frontal $30 \%$, partial $26 \%$, and occipital $8 \%$. $^{10}$ Ashraf et al. reported location wise chances of brain abscess $46 \%$ in frontal, $28 \%$ in temporoparietal, $8 \%$ in occipital region and $8 \%$ at multiple areas. ${ }^{15}$

Culture was positive in $12(48 \%)$ and no growth obtained in $13(52 \%)$ cases. Ashraf et al. reported culture positive in $36 \%$ and negative in $64 \%{ }^{15}$ Streptococcus milleri was most common organism followed by staphylococcus aureus and E.coli. Streptococcus milleri was the commonest organism isolated from pus in other studies conducted by Ashraf et al. ${ }^{15}$ Atiq et al. ${ }^{16}$ and Sineviratne et al. ${ }^{17}$ Staphylococcus aureus was the commonest organism reported by Bhand et al. ${ }^{18}$ Muzumdar D et al. reported bacteroides as common microorganism followed by peptostreptococcus and streptococcus. ${ }^{9}$ Tonon et al. reported streptococci viridians followed by staphylococcus aureus, gram-negative bacilli, and anaerobes as common causative microorganism. ${ }^{10}$ 
Pus was aspirated by an appropriate size needle in $4(16 \%)$ patients within one year of age having open anterior fontanelle and pus located in frontal and temporoparietal region. Burr hole was performed and pus aspirated with brain cannula in $15(60 \%)$ and craniotomy performed for excision of thick pus in $6(24 \%)$. Same surgical intervention protocol is mentioned by Brouwer $\mathrm{MC}$ et al. ${ }^{4}$ Ranjith et al. ${ }^{7}$ and Muzumdar D et al. ${ }^{9}$ Eva Tonon et al. mentioned craniotomy for brain abscess as obsolete procedure. ${ }^{10}$

Three (12\%) patients expired, two after craniotomy and one after aspiration of abscess. With improvement in diagnostic technique new antibiotic and surgical techniques outcome of management of disease has improved and mortality has been reduced..$^{7,9}$ Eva Tonon et al. ${ }^{10}$ mentioned $8 \%$ mortality, Prusty ${ }^{19}$ reported it up to $17 \%$ and Ashraf et al. found no mortality in early postoperative period after aspiration of brain abscess. ${ }^{15}$ Outcome of management of brain abscess was assessed clinically and with follow up CT scan in every patient and same protocol is mentioned by Nathoo et al. ${ }^{20}$ Follow up ultrasonography through open anterior fontanelle in four infants was performed in this study. It can be performed through burr hole made for initial surgery as reported by Hayashi et al. ${ }^{21}$

Limitations of the study: It includes a small sample size. Further studies with a larger sample size are suggested.

\section{CONCLUSIONS}

As paranasal sinusitis and chronic otitis media are common in developing countries and these are common predisposing factors of brain abscess so early and complete treatment of these causes may reduce chances of brain abscess to develop. Early diagnosis of suspected cases of brain abscess and prompt empirical antibiotic therapy should be started immediately in every case of brain abscess even in cerebritis stage. Small abscess less than $2 \mathrm{~cm}$ can be managed without surgical intervention. Minimal invasive surgical procedure of aspiration is effective and relatively safe. Craniotomy and excision of abscess is preferred in case of thick, septated and multiloculated pus.

\section{REFERENCES}

1. Takeshita M, Kagawa M, Yato S. Cerebral abscess. Greenberg M S. Handbook of neurosurgery. $7^{\text {th }}$ edition Newyork: Thieme; 2006: pp.350-356.

2. Helweg-Larsen J, Astradsson A, Richhall H, Erdal J, Laursen A, Brennum J. Pyogenic brain abscess, a 15 year survey. BMC Infect Dis. 2012;12:332. doi: 10.1186/1471-2334-12-332
3. Brouwer MC, Countinho JM, van de Beek D. Clinical characteristics and out-come of brain abscess: Systematic review and meta-analysis. Neurology. 2014;82:806-813.

4. Brouwer MC, Tunkel AR, McKhann GM, van de Beek D Brain abscess: N Engl J Med. 2014;371(5):447-56. doi: 10.1056/ NEJMra1301635

5. Reddy JS, Mishra AM, Behari S, Husain M, Gupta V, Rastogi M, et al The role of diffusion-weighted imaging in the differential diagnosis of intracranial cystic mass lesions: A report of 147 lesions. Surg Neurol. 2006;66:246-250. doi: 10.1016/j.surneu.2006.03.032

6. Martin-Canal G, Saavedra A, Asensi JM, Suarez-Zarracina T, Rodriguez-Guardado A, Bustillo E, et al. Meropenen monotherapy is as effective as and safer than imipenem to treat brain abscesses. Int J Antimicrob Agents. 2010;35:301-304. doi: 10.1016/j. ijantimicag.2009.11.012

7. Ranjith KM, Vedantam R. Management of brain abscess: an overview. Neurosurg Focus. 2008;24(6):E3.

8. Tony AT, Ranjith KM. Brain abscess: Diagnosis and management. Neurosurg Focus CMI. 2015;13(4):55-61.

9. Muzumdar D, Jhawar S, Goel A. Brain Abscess: An overview. Int J Surg. 2011;9(2):136-144. doi: 10.1016/j.ijsu.2010.11.005

10. Tonon E, Scotton PG, Gallucci M, Vaglia A. Brain abscess: Clinical aspects of 100 patients. Int J Infect Dis. 2006;10(2):103-109. doi: 10.1016/j.ijid.2005.04.003

11. Chuang MJ, Chang WN, Chang HW, Lin WC, Tsai NW, Hsieh MJ, et al. Predictors and long-term outcome of seizures after bacterial brain abscess. J Neurol Neurosurg Psychiatry. 2010;81(8):913-917. doi: 10.1136/jnnp.2009.195073

12. Stanescu GL, Nedelcuta RM, Dop D, Diaconu R, Calin G, Niculescu EC, et al. Brain abscess of unknown etiology. Rom J Morphol Embryol. 2015;56(3):1201-1204.

13. Nelson CA, Zunt JR. Tuberculosis of the central nervous system in immune-compromised patients: HIV infection and solid organ transplant recipients. Clin Infect Dis. 2011;53:915-926. doi: $10.1093 / \mathrm{cid} / \operatorname{cir} 508$

14. Yang KY, Chang WN, Ho JT, Wang HC, Lu CH. Post neurosurgical nosocomial bacterial brain abscess in adults. Infection. 2006;34(5):247251. doi: $10.1007 /$ s15010-006-5607-5

15. Ashraf M, Shakeel A, Shafiq A Hussain M. Burr hole aspiration of brain abscess in children with cyanotic heart disease. J Coll Physicians Surg Pak. 2017;27(8):483-485.

16. Atiq M, Ahmed US, Allana SS, Chishti KN. Brain abscess in children India J Pediatr. 2006;73(5):401-404. doi: 10.1007/BF02758560.

17. Seneviratne Rde S, Navasivayam P, Perera S, Wickremasinghe RS. Microbiology of brain abscess in a neurosurgical unit of the National Hospital of Sri Lanka. Ceylon Med J. 2003;48(1):14-16.

18. Bhand AA. Brain abscess-diagnosis and management. J Coll Physicians Surg Pak. 2004;14(7):407-410. doi: 07.2004/JCPSP.407410

19. Prusty GK. Brain Abscesses in cyanotic heart disease. Indian J Pediatr. 1993;60(1):43-51.

20. Nathoo N, Nadvi SS, Narotam PK, van Dellen JR. Brain abscess: Management and outcome analysis of a computed tomography era experience with 973 patients. World Neurosurg. 2011;75(5-6):716726. doi: 10.1016/j.wneu.2010.11.043

21. Hayashi K, Matsuo T, Suyama K, Nagata I. Usefulness of ultrasonography with a burr-hole transducer during surgery through a burr hole. Neurol Med Chir (Tokyo). 2012;52(3):165-168. doi: $10.2176 /$ nmc.52.165

\section{Authors' Contribution:}

IUK: conceived, designed and did statistical analysis \& editing of manuscript and takes the responsibility and is accountable for all aspects of the work in ensuring that questions related to the accuracy or integrity of any part of the work are appropriately investigated and resolved.

AL: did data collection and manuscript writing.

MA: did data analysis and manuscript writing.

MKC: Manuscript writing.

SS: Manuscript writing and proof reading. 